\title{
A Meta-Analysis about the Screening Role of Pulse Oximetry for Congenital Heart Disease
}

\author{
Caiju Du, ${ }^{1}$ Dianmei Liu, ${ }^{2}$ Guojing Liu, ${ }^{3}$ and Huaixin Wang ${ }^{4}$ \\ ${ }^{1}$ Cardiovascular Surgery, Affiliated Hospital of Weifang Medical University, 2428 Yuhe Road, Kuiwen District, \\ Weifang, Shandong Province 261031, China \\ ${ }^{2}$ Imaging Center, Affiliated Hospital of Weifang Medical University, 2428 Yuhe Road, Kuiwen District, \\ Weifang, Shandong Province 261031, China \\ ${ }^{3}$ Operating Room, Affiliated Hospital of Weifang Medical University, 2428 Yuhe Road, Kuiwen District, \\ Weifang, Shandong Province 261031, China \\ ${ }^{4}$ Emergency Department, Yidu Central Affiliated Hospital of Weifang Medical University, 4138 Linglong South Road, \\ Qingzhou, Shandong Province 262550, China
}

Correspondence should be addressed to Dianmei Liu; dianml05@126.com

Received 8 June 2017; Accepted 20 November 2017; Published 10 December 2017

Academic Editor: Vladimiro Vida

Copyright (c) 2017 Caiju Du et al. This is an open access article distributed under the Creative Commons Attribution License, which permits unrestricted use, distribution, and reproduction in any medium, provided the original work is properly cited.

Objective. The opinions about the application of pulse oximetry in diagnosis of congenital heart disease (CHD) were debatable. We performed this meta-analysis to confirm the diagnostic role of pulse oximetry screening for CHD. Methods. Relevant articles were searched in the databases of Pubmed, Embase, Google Scholar, and Chinese National Knowledge Infrastructure (CNKI) up to April 2017. Data was processed in the MetaDiSc 1.4 software. Pooled sensitivity and specificity with $95 \%$ confidence interval ( $95 \%$ CI) were calculated to explain the diagnostic role of pulse oximetry screening for CHD. $I^{2} \geqslant 50 \%$ or $p<0.05$ indicated significant heterogeneity. Area under curve (AUC) of summary receiver operating characteristics (SROC) was calculated to assess its diagnostic accuracy. The robustness of overall results was evaluated by sensitivity analysis. Publication bias was evaluated by Deek's funnel plot. Results. 22 eligible articles were selected. Pooled sensitivity and specificity were $0.69(0.67-0.72)$ and 0.99 (0.990.99), respectively. The corresponding AUC was 0.9407 , suggesting high diagnostic accuracy of pulse oximetry screening for CHD. Sensitivity analysis demonstrated that the pooled results were robust. Deek's funnel plot seemed to be symmetrical. Conclusions. Pulse oximetry screening could be used to diagnose CHD. It shows high diagnosis specificity and accuracy.

\section{Introduction}

Congenital heart disease (CHD) is regarded as a main cause of infant death, with an incidence of 8 in every 1000 live births [1]. It needs invasive intervention during the neonatal period and these neonates suffering this disease benefit most from early detection [2]. Prenatal diagnosis just picks up $<50 \%$ of all cases [3-6]. Routine neonatal inspection fails to detect above than $50 \%$ of CHD infants. More than $55 \%$ of neonates show no murmur symptom in the nursery, and $\leqslant 82 \%$ of them are discharged before diagnosis results are obtained [7]. Early diagnosis of $\mathrm{CHD}$ is crucial since the delayed diagnosis results in cardiovascular collapse, cardiac failure, and death, whereas early diagnosis during the first few days of life is difficult. Therefore, an effective screening program for $\mathrm{CHD}$ is necessary.

In recent years, pulse oximetry has been suggested as a diagnosis tool for CHD [8-10]. It is productive in the detection of $\mathrm{CHD}$ before discharge and could decrease missed cases to $4 \%[11,12]$. Some states of United States have made screening of pulse oximetry for CHD mandatory before discharge in hospital. Pulse oximetry can detect mild hypoxemia, which is common feature for many forms of CHD. It could recognize the cases that are not recognized by clinical examination [13]. Since the introduction of pulse oximetry to screen CHD in 1995, many studies have focused on the subject [14-17]. Despite the fact that there were differences in screening time, cut-off values, target lesions, and others 


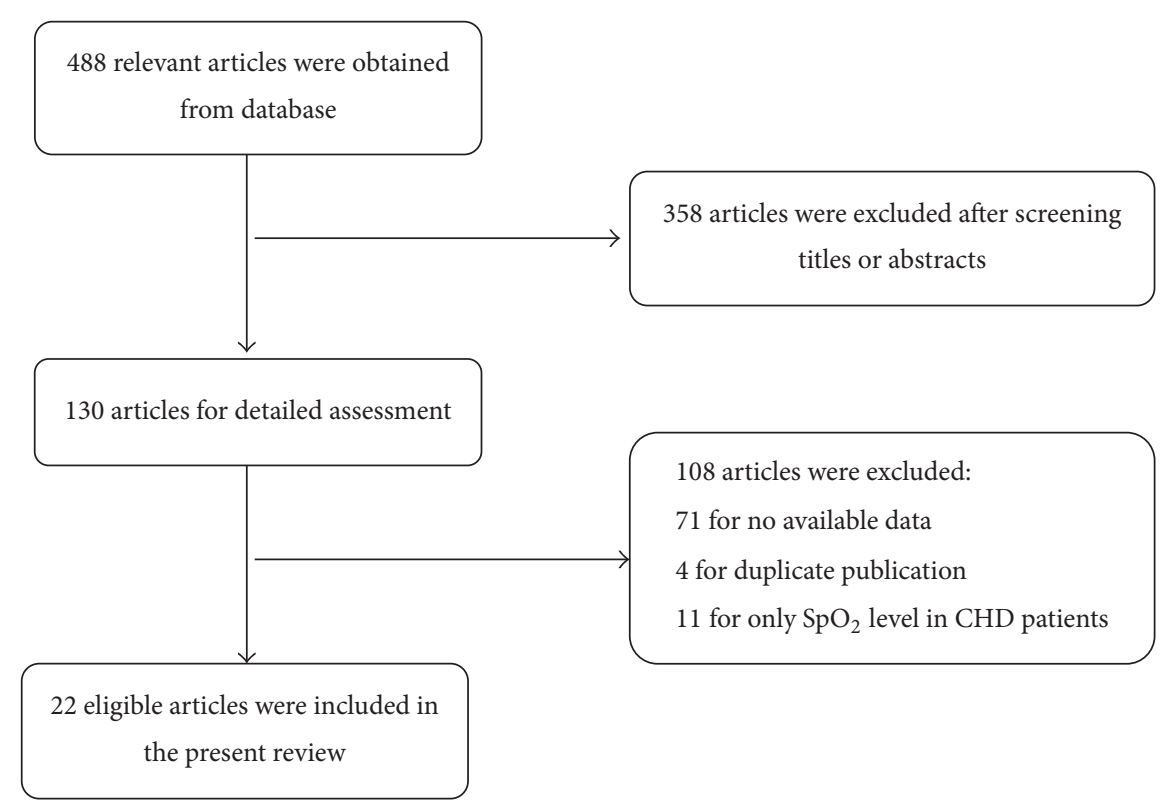

FIGURE 1: Flow chart of articles selection. 22 articles were selected for meta-analysis.

among the relevant studies, the opinion is consistent that pulse oximetry screening is a useful diagnostic method of CHD. However, existing data is still insufficient to initiate a recommendation for application of pulse oximetry in routine care.

This present meta-analysis was performed to confirm the diagnostic role of pulse oximetry for $\mathrm{CHD}$. The obtained results contribute to clinical application of pulse oximetry for diagnosing CHD.

\section{Materials and Methods}

2.1. Search Strategy. We searched the relevant articles on the databases of Pubmed, Embase, Google Scholar, and Chinese National Knowledge Infrastructure (CNKI) up to April 2017. The following keywords were used: pulse oximetry OR SpO2 AND congenital heart disease OR CHD. The references' lists of obtained articles were manually searched for eligible studies. No language restriction was applied. The studies published in abstract were not considered.

2.2. Article Selection. These obtained articles were selected according to inclusion criteria. The criteria were as follows: (a) $\mathrm{SpO}_{2}$ was assessed with pulse oximetry; (b) $\mathrm{SpO}_{2}$ was used to detect CHD subjects; (c) true positive (TP), false positive (FP), true negative (TN), and false negative (FN) or other data available for calculating them were reported. The review article, abstract article, and case reports were removed from the present analysis.

2.3. Data Extraction. Two authors were responsible for extracting data. The data included name of first author, year of publication, country, number of patients and healthy controls, screening time, screening limb, TP, FP, TN, and FN.
The inconsistent opinion was solved with a discussion with the third author.

2.4. Statistical Analysis. Data was processed in the MetaDiSc 1.4 software. Deek's funnel plot was obtained with Stata 12.0 software. Summary sensitivity and specificity along with $95 \%$ confidence interval (95\% CI) were adopted to confirm the diagnostic role of pulse oximetry screening for CHD. $I^{2} \geqslant$ $50 \%$ or $p<0.05$ indicated significant heterogeneity. Area under curve (AUC) of summary receiver operating characteristics (SROC) was calculated to assess the diagnostic accuracy of $\mathrm{SpO}_{2}$. The robustness of overall results was evaluated by sensitivity analysis. Publication bias was evaluated by Deek's funnel plot.

\section{Results}

3.1. Selection Process of Eligible Articles. A total of 488 relevant articles were obtained after search on the databases. The titles and abstracts were screened and 358 articles were excluded. The remaining 130 articles were provided detailed assessment and 108 articles were excluded for no available data, duplicate publication, and only $\mathrm{SpO}_{2}$ level in CHD patients. Finally, 22 eligible articles were included in the present meta-analysis $[8,10-12,14-31]$. The detailed selection process was shown in Figure 1. Basic information of each study was listed in Table 1.

3.2. Diagnostic Role of Pulse Oximetry Screening for CHD. Pulse oximetry screening showed high specificity in detecting CHD (specificity: 0.99), while having relatively low sensitivity (0.69) (Figure 2). In the analyses of sensitivity and specificity, we observed significant heterogeneity (sensitivity: $p=$ $0.0000, I^{2}=89.6 \%$; specificity: $\left.p=0.0000, I^{2}=99.9 \%\right)$. The corresponding AUC was 0.9407, suggesting high diagnostic accuracy of pulse oximetry screening for CHD (Figure 3). 
TABLE 1: Basic information of included studies.

\begin{tabular}{|c|c|c|c|c|}
\hline Author & Year & Country & Limb & Test timing, $\mathrm{h}$ \\
\hline Arlettaz et al. & 2006 & Switzerland & Right or left foot & $6-12$ \\
\hline Bakr and Habib & 2005 & Egypt & Right upper and lower limbs & 31.7 (average) \\
\hline de Wahl Granelli et al. & 2005 & Sweden & Right hand and one foot & $12-48$ \\
\hline Hoke et al. & 2002 & America & Right arm and either leg & 24 (average) \\
\hline Koppel et al. & 2003 & America & - & $>24$ \\
\hline Richmond et al. & 2002 & UK & One or other foot & $>2$ \\
\hline Rosati et al. & 2005 & Italy & - & $72 \mathrm{~h}$ (median) \\
\hline Zhao et al. & 2014 & China & Both on the right hand and on either foot & $6-72$ \\
\hline Riede et al. & 2010 & Germany & Foot & $24-72$ \\
\hline Mathur et al. & 2015 & India & Right upper limb and either foot & 72 (median) \\
\hline Hu et al. & 2016 & China & Right hand and either foot & 25 (median) \\
\hline Jones et al. & 2016 & UK & - & $<24$ \\
\hline Ozalkaya et al. & 2016 & Turkey & Lower and right upper extremity & $>24$ \\
\hline Jawin et al. & 2015 & Malaysia & Left foot & 20 (median) \\
\hline Ewer et al. & 2011 & UK & Right hand and either foot & In the first few hours \\
\hline Taksande et al. & 2013 & India & All the four limbs & Within the first 4 hours \\
\hline van Niekerk et al. & 2016 & South Africa & - & $60($ median $)$ \\
\hline de-Wahl Granelli et al. & 2009 & Sweden & Right hand and either foot & 38 (median) \\
\hline Meberg et al. & 2008 & Norway & Foot & $<12$ \\
\hline Mo et al. & 2015 & China & Right hand and either foot & $>24$ \\
\hline Jia et al. & 2016 & China & Right hand & 24 \\
\hline Lu et al. & 2016 & China & Right hand and right foot & $>24$ \\
\hline
\end{tabular}

Note. - indicates that the information was not mentioned in the article.


FIGURE 2: Diagnostic sensitivity and specificity of pulse oximetry screening for congenital heart disease (CHD). The sensitivity and specificity were $0.69(0.67-0.72)$ and 0.99 (0.99-0.99), respectively.

3.3. Sensitivity Analysis and Publication Bias Detection. Robustness of pooled results was assessed by sensitivity analysis by deleting one study each time. The analysis indicated that the pooled results were robust. Deek's funnel plot was used to assess publication bias. The funnel plot seemed to be symmetrical (Figure 4).

\section{Discussion}

CHD is a series of heterogeneous disorders that catheter intervention or surgery is mandatory to achieve patients' survival. Clinical examination shows limitation in detecting all forms of CHD [32, 33]. Heart murmurs, one of the hallmarks 


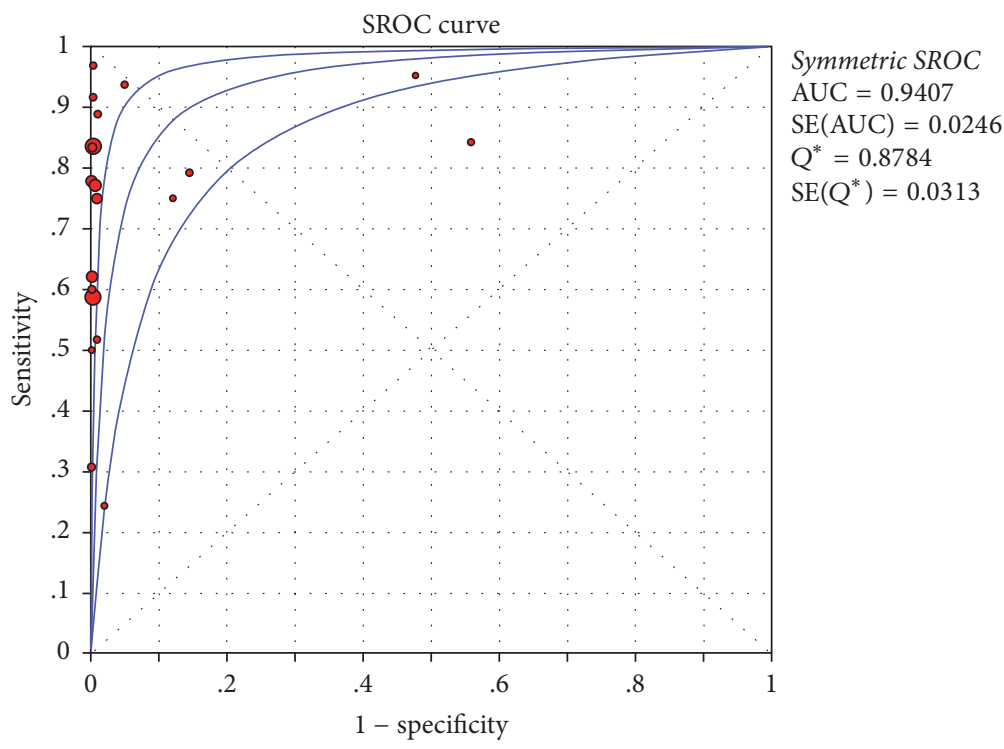

FIgURE 3: Area under curve (AUC) of SROC curve. AUC was 0.9407.

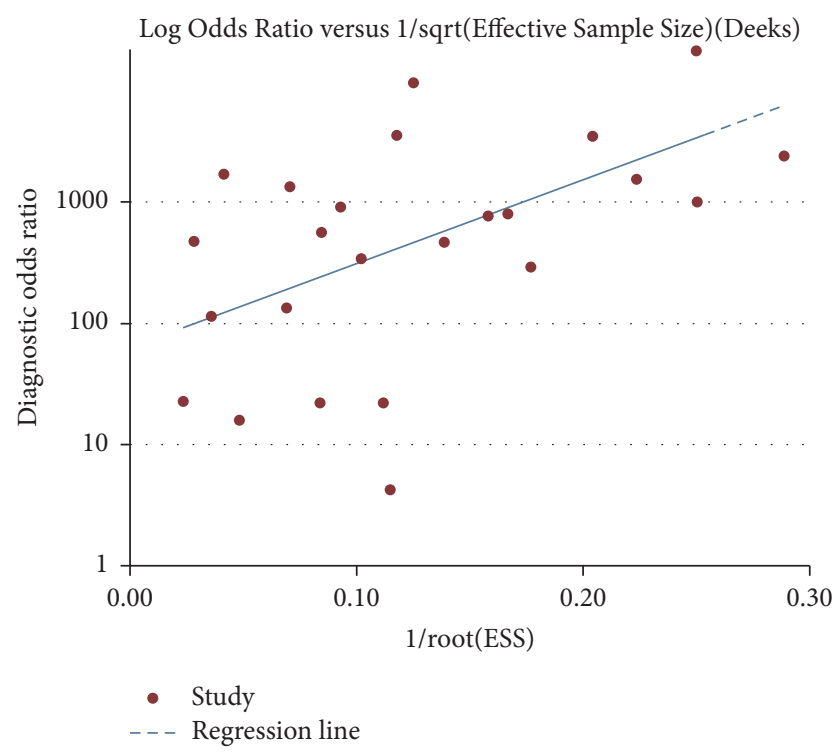

Figure 4: Deek's funnel plot. We found no significant publication bias in the present meta-analysis.

of $\mathrm{CHD}$, may be misleading or absent due to the reduced ventricular function, prolonged decline of pulmonary vascular resistance, and underlying anatomy. Although prenatal diagnosis is widely applied, a large proportion of $\mathrm{CHD}$ neonates are still not diagnosed before being discharged and after birth [34, 35], which may be strengthened by earlier discharge and certain postnatal care [36]. It has been thought that the application of prenatal ultrasound, clinical observation, and physical examination may be sufficient for early diagnosis of CHD [37]. This opinion may be true under specific circumstances; however, the prerequisites possibly do not exist in the majority of hospitals. Thus, a broad consensus that efficient diagnostic tool for CHD is urgently needed has been achieved.

Pulse oximetry is an accurate and noninvasive test for quantification of hypoxaemia. The application of this method for diagnosing CHD is based on the theory that undetectable hypoxaemia in clinic exists in most life-threatening cases. Pulse oximetry screening for CHD has gained more attention over the last decade. It has been demonstrated to be costeffective and acceptable to the mothers $[38,39]$. The existing protocol of pulse oximetry to detect CHD is restricted to 24 to 48 hours of age for neonates in well infant nursery [40].

Our meta-analysis included as many eligible articles as possible via systematic search. These obtained articles were selected carefully according to inclusion criteria. Moreover, the quality of included articles was high. Besides, the results were based on 22 eligible studies involving both Western and Asian countries. Therefore, our results were reliable. The pooled results suggested that combined sensitivity, specificity, and AUC were 0.69 (0.67-0.72), 0.99 (0.99-0.99), and 0.9407, respectively, which is similar to the previous meta-analysis [41].

Among the included articles, the results showed great differences. In the study by Mathur et al., pulse oximetry readings were taken at admission from 950 neonates and the diagnostic sensitivity, specificity, positive predictive value, and negative predictive value were $95.2 \%, 52.4 \%, 9.5 \%$, and $99.5 \%$. The diagnostic specificity was poor. Similarly, $\mathrm{Hu}$ et al. reported that diagnostic specificity of pulse oximetry screening for CHD was just $44.22 \%$. Meanwhile, Niekerk et al. reported that the diagnostic sensitivity of pulse oximetry was merely $50 \%$, while the specificity was $99.9 \%$. On the contrary, Arlettaz et al. investigated the contribution of pulse oximetry to the early detection of CHD in newborns and found that the sensitivity and specificity were $100 \%$ and $99.7 \%$, respectively. In the study of Jones et al., the estimated sensitivity and specificity were $100 \%$ and $99.8 \%$ of pulse oximetry screening 
for diagnosing CHD. Thus, the conclusion of our analysis is significant to confirm the diagnostic role of pulse oximetry screening.

However, we must acknowledge that there were limitations in the present meta-analysis. First, cut-off value, diagnosis criteria, target location, and test timing of pulse oximetry were inconsistent among the included studies, which might affect the diagnostic accuracy of pulse oximetry screening. And the significant heterogeneity in the present analysis might result from these variances. Besides, the difference in the severity of CHD also might influence the accuracy of pulse oximetry screening.

\section{Conclusion}

In conclusion, pulse oximetry screening may serve as a valuable diagnostic tool with high accuracy for $\mathrm{CHD}$. The diagnostic sensitivity and specificity are $0.69(0.67-0.72)$ and 0.99 (0.99-0.99), respectively.

\section{Conflicts of Interest}

The authors declare that there are no conflicts of interest regarding the publication of this paper.

\section{Authors' Contributions}

Caiju Du and Dianmei Liu were involved in the conception and design, or analysis and interpretation of the data; Guojing Liu was involved in the interpretation of the data; Huaixin Wang revised the manuscript critically for intellectual content. All the authors approve the final version to be published and agree to be accountable for all aspects of the work.

\section{Acknowledgments}

This work was supported by the Science and Technology Development Plan Project in Medicine and Health Care of Shandong Province under Grant no. 2015WS0044.

\section{References}

[1] R. M. Payne, M. C. Johnson, J. W. Grant, and A. W. Strauss, "Toward a molecular understanding of congenital heart disease," Circulation, vol. 91, no. 2, pp. 494-504, 1995.

[2] R.-K. R. Chang, M. Gurvitz, and S. Rodriguez, "Missed diagnosis of critical congenital heart disease," JAMA Pediatrics, vol. 162, no. 10, pp. 969-974, 2008.

[3] M. Abu-Harb, J. Wyllie, E. Hey, S. Richmond, and C. Wren, "Presentation of obstructive left heart malformations in infancy.", Archives of Disease in Childhood - Fetal and Neonatal Edition, vol. 71, no. 3, pp. F179-F183, 1994.

[4] C. Bull, "Current and potential impact of fetal diagnosis on prevalence and spectrum of serious congenital heart disease at term in the UK," The Lancet, vol. 354, no. 9186, pp. 1242-1247, 1999.

[5] Z.-D. Du, N. Roguin, and M. Barak, "Clinical and echocardiographic evaluation of neonates with heart murmurs," Acta Paediatrica, vol. 86, no. 7, pp. 752-756, 1997.
[6] J. Gregory, A. Emslie, J. Wyllie, and C. Wren, "Examination for cardiac malformations at six weeks of age," $A D C$ - Fetal and Neonatal Edition, vol. 80, no. 1, pp. F46-F48, 1999.

[7] B. Varan, K. Tokel, and G. Yilmaz, "Malnutrition and growth failure in cyanotic and acyanotic congenital heart disease with and without pulmonary hypertension," Archives of Disease in Childhood, vol. 81, no. 1, pp. 49-52, 1999.

[8] R. I. Koppel, C. M. Druschel, T. Carter et al., "Effectiveness of pulse oximetry screening for congenital heart disease in asymptomatic newborns," Pediatrics, vol. 111, no. 3, pp. 451-455, 2003.

[9] J. Reich, S. Miller, and B. Brogdon, "The use of pulse oximetry to detect congenital heart disease," ACC Current Journal Review, vol. 12, no. 4, p. 92, 2003.

[10] S. Richmond, G. Reay, and M. Abu Harb, "Routine pulse oximetry in the asymptomatic newborn," ADC - Fetal and Neonatal Edition, vol. 87, no. 2, pp. F83-F88, 2002.

[11] A. de-Wahl Granelli, M. Wennergren, K. Sandberg et al., "Impact of pulse oximetry screening on the detection of duct dependent congenital heart disease: a Swedish prospective screening study in 39,821 newborns.," BMJ (Clinical research ed.), vol. 338, p. a3037, 2009.

[12] F. T. Riede, C. Wörner, I. Dähnert, A. Möckel, M. Kostelka, and P. Schneider, "Effectiveness of neonatal pulse oximetry screening for detection of critical congenital heart disease in daily clinical routine-results from a prospective multicenter study," European Journal of Pediatrics, vol. 169, no. 8, pp. 975-981, 2010.

[13] C. P. F. O’Donnell, C. Omar, F. Kamlin, P. G. Davis, J. B. Carlin, and C. J. Morley, "Clinical assessment of infant colour at delivery," ADC - Fetal and Neonatal Edition, vol. 92, no. 6, pp. F465-F467, 2007.

[14] R. Arlettaz, A. S. Bauschatz, M. Mönkhoff, B. Essers, and U. Bauersfeld, "The contribution of pulse oximetry to the early detection of congenital heart disease in newborns," European Journal of Pediatrics, vol. 165, no. 2, pp. 94-98, 2006.

[15] A. F. Bakr and H. S. Habib, "Combining pulse oximetry and clinical examination in screening for congenital heart disease," Pediatric Cardiology, vol. 26, no. 6, pp. 832-835, 2005.

[16] T. R. Hoke, P. K. Donohue, P. K. Bawa et al., "Oxygen saturation as a screening test for critical congenital heart disease: A preliminary study," Pediatric Cardiology, vol. 23, no. 4, pp. 403409, 2002.

[17] E. Rosati, G. Chitano, L. Dipaola, C. De Felice, and G. Latini, "Indications and limitations for a neonatal pulse oximetry screening of critical congenital heart disease," Journal of Perinatal Medicine, vol. 33, no. 5, pp. 455-457, 2005.

[18] A. de Wahl Granelli, M. Mellander, J. Sunnegårdh, K. Sandberg, and I. Ostman-Smith, "Screening for duct-dependant congenital heart disease with pulse oximetry: a critical evaluation of strategies to maximize sensitivity." Acta paediatrica (Oslo, Norway: 1992), vol. 94, no. 11, pp. 1590-1596, 2005.

[19] Q. M. Zhao, X. J. Ma, X. L. Ge et al., "Pulse oximetry with clinical assessment to screen for congenital heart disease in neonates in China: a prospective study," Lancet, vol. 384, no. 9945, pp. 747754, 2014.

[20] N. B. Mathur, A. Gupta, and S. Kurien, "Pulse oximetry screening to detect cyanotic congenital heart disease in sick neonates in a neonatal intensive care unit," Indian Pediatrics, vol. 52, no. 9, pp. 769-772, 2015.

[21] X.-J. Hu, Q.-M. Zhao, X.-J. Ma et al., "Pulse oximetry could significantly enhance the early detection of critical congenital heart disease in neonatal intensive care units," Acta Paediatrica, vol. 105, no. 11, pp. e499-e505, 2016. 
[22] A. J. Jones, C. Howarth, R. Nicholl, E. Mat-Ali, and R. Knowles, "The impact and efficacy of routine pulse oximetry screening for CHD in a local hospital," Cardiology in the Young, vol. 26, no. 7, pp. 1397-1405, 2016.

[23] E. Özalkaya, A. Akdağ, I. Şen, E. Cömert, and H. Melek Yaren, "Early screening for critical congenital heart defects in asymptomatic newborns in Bursa province," The Journal of MaternalFetal and Neonatal Medicine, vol. 29, no. 7, pp. 1105-1107, 2016.

[24] V. Jawin, H.-L. Ang, A. Omar, and M.-K. Thong, "Beyond critical congenital heart disease: Newborn screening using pulse oximetry for neonatal sepsis and respiratory diseases in a Middle-Income Country," PLoS ONE, vol. 10, no. 9, Article ID e0137580, 2015.

[25] A. K. Ewer, L. J. Middleton, A. T. Furmston et al., "Pulse oximetry screening for congenital heart defects in newborn infants (PulseOx): A test accuracy study," The Lancet, vol. 378, no. 9793, pp. 785-794, 2011.

[26] A. M. Taksande, B. Lakhkar, and A. Gadekar, "Accuracy of pulse oximetry screening for detecting critical congenital heart disease in the newborns in rural hospital of Central India," Images Paediatr Cardiol, vol. 15, no. 4, pp. 5-10, 2013.

[27] A. M. van Niekerk, R. M. Cullis, L. L. Linley, and L. Zühlke, "Feasibility of pulse oximetry pre-discharge screening implementation for detecting critical congenital heart lesions in newborns in a secondary-level maternity hospital in the Western Cape, South Africa: The 'POPSICLe' study,' South African Medical Journal, vol. 106, no. 8, pp. 817-821, 2016.

[28] A. Meberg, S. Brügmann-Pieper, R. Due Jr. et al., "First Day of Life Pulse Oximetry Screening to Detect Congenital Heart Defects," Journal of Pediatrics, vol. 152, no. 6, pp. 761-765, 2008.

[29] Z. D. Mo, X. L. Guo, and W. J. Wang, "Effectiveness of pulse oximetry for the screening of congenital heart disease in newborn infants," Chinese Journal of Neonatology, vol. 30, no. 5, pp. 343-346, 2015.

[30] A. Q. Jia, J. H. Wu, and A. Y. Guo, "Analysis of intervals of pulse oximetry in congenital heart disease screening," J Clin Pediatr, vol. 34, no. 5, pp. 357-359, 2016.

[31] X. Y. Lu and Y. Li, "Comparison between pulse oximetry and traditional method in screening congenital heart disease," Chinese Journal of Clinical Rational Drug Use, vol. 9, no. 10A, pp. 152-153, 2016.

[32] S. B. Ainsworth, J. P. Wyllie, and C. Wren, "Prevalence and clinical significance of cardiac murmurs in neonates," $A D C$ Fetal and Neonatal Edition, vol. 80, no. 1, pp. F43-F45, 1999.

[33] C. Wren, S. Richmond, and L. Donaldson, "Presentation of congenital heart disease in infancy: Implications for routine examination," ADC - Fetal and Neonatal Edition, vol. 80, no. 1, pp. F49-F53, 1999.

[34] R.-K. R. Chang, S. Rodriguez, and T. S. Klitzner, "Screening newborns for congenital heart disease with pulse oximetry: Survey of pediatric cardiologists," Pediatric Cardiology, vol. 30, no. 1, pp. 20-25, 2009.

[35] C. Wren, Z. Reinhardt, and K. Khawaja, "Twenty-year trends in diagnosis of life-threatening neonatal cardiovascular malformations," ADC - Fetal and Neonatal Edition, vol. 93, no. 1, pp. F33-F35, 2008.

[36] M. Mellander and J. Sunnegårdh, "Failure to diagnose critical heart malformations in newborns before discharge - An increasing problem?" Acta Paediatrica, vol. 95, no. 4, pp. 407413, 2006.

[37] D. M. Sendelbach, G. L. Jackson, S. S. Lai, D. E. Fixler, E. K. Stehel, and W. D. Engle, "Pulse oximetry screening at 4 hours of age to detect critical congenital heart defects," Pediatrics, vol. 122, no. 4, pp. e815-e820, 2008.

[38] A. Ewer, A. Furmston, L. Middleton et al., "Pulse oximetry as a screening test for congenital heart defects in newborn infants: a test accuracy study with evaluation of acceptability and costeffectiveness.", Health Technology Assessment, vol. 16, no. 2, 2012.

[39] R. Powell, H. M. Pattison, A. Bhoyar et al., "Pulse oximetry screening for congenital heart defects in newborn infants: An evaluation of acceptability to mothers," ADC - Fetal and Neonatal Edition, vol. 98, no. 1, pp. F59-F63, 2013.

[40] A. R. Kemper, W. T. Mahle, G. R. Martin et al., "Strategies for implementing screening for critical congenital heart disease," Pediatrics, vol. 128, no. 5, pp. e1259-e1267, 2011.

[41] S. Thangaratinam, K. Brown, J. Zamora, K. S. Khan, and A. K. Ewer, "Pulse oximetry screening for critical congenital heart defects in asymptomatic newborn babies: A systematic review and meta-analysis," The Lancet, vol. 379 , no. 9835, pp. 2459$2464,2012$. 




The Scientific World Journal
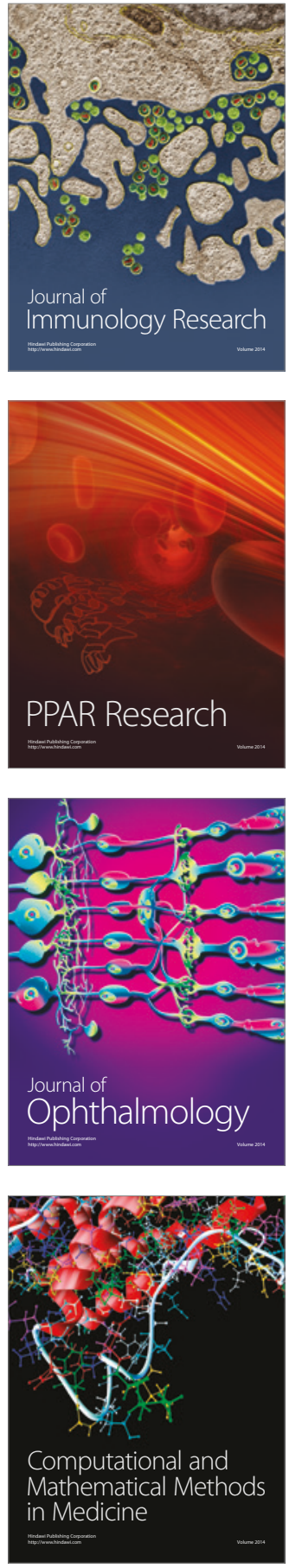

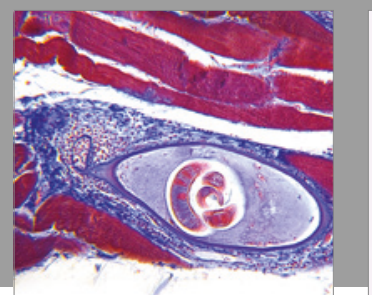

Gastroenterology Research and Practice
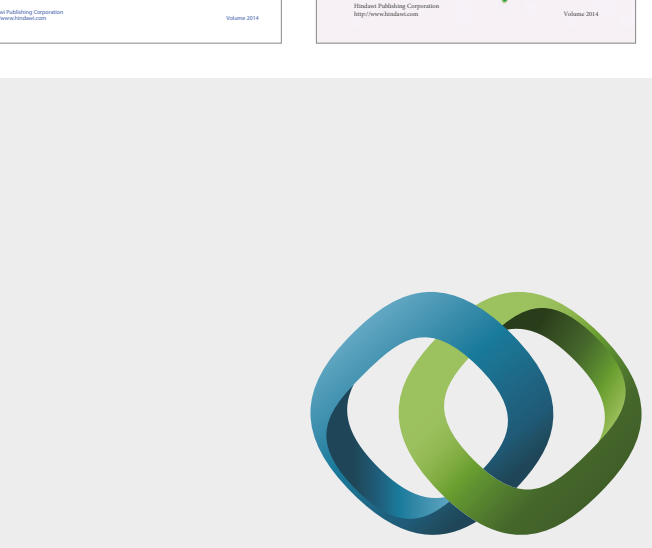

\section{Hindawi}

Submit your manuscripts at

https://www.hindawi.com
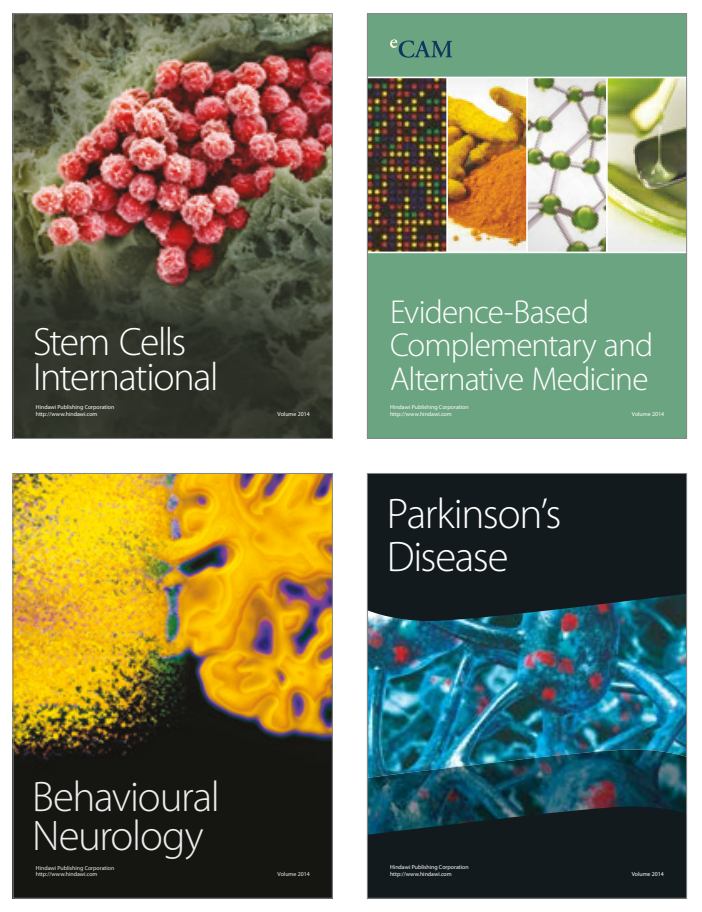
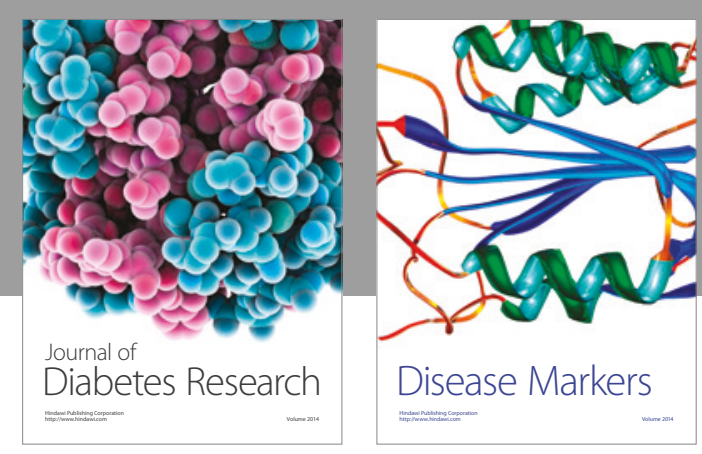

Disease Markers
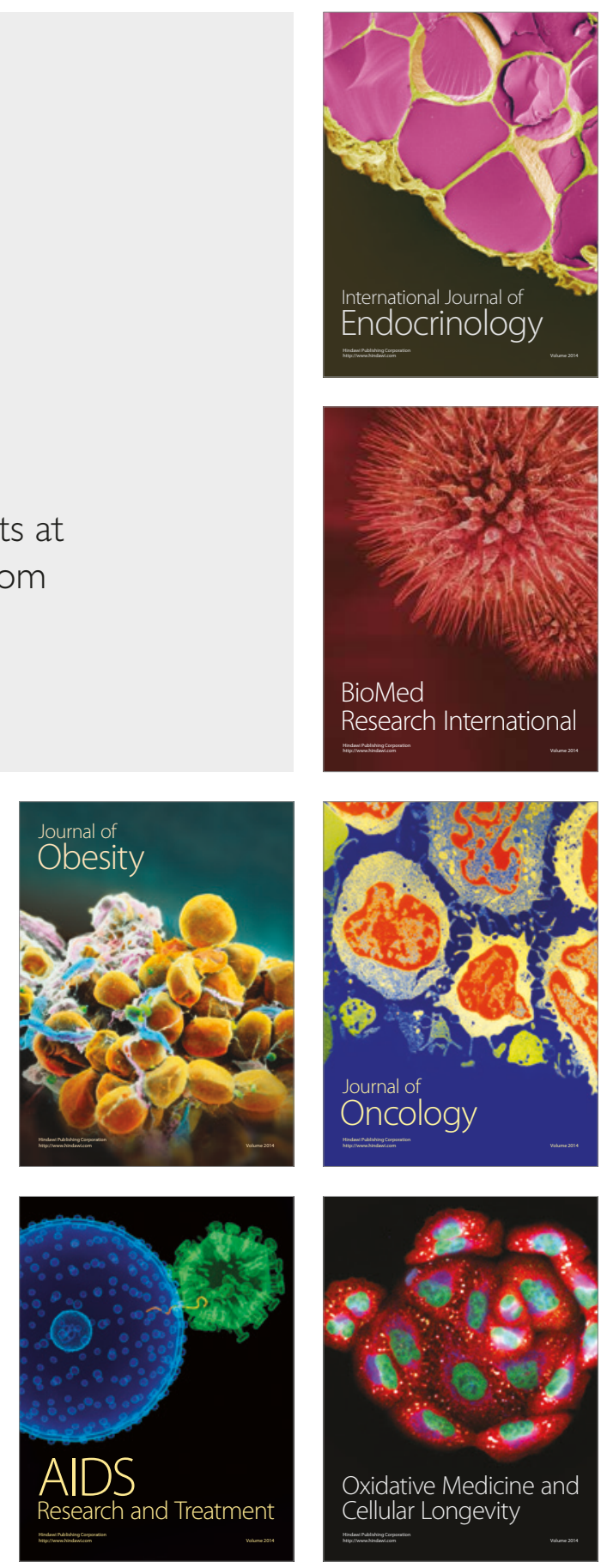